\title{
Mosquito Larvicidal Activity of the Essential Oils of Erechtites Species Growing Wild in Vietnam
}

\author{
Nguyen Huy Hung $\left.{ }^{1}{ }^{(}\right)$, Prabodh Satyal ${ }^{2}$, Ho Viet Hieu ${ }^{3, *}{ }^{\circledR}$, Nguyen Thi Hong Chuong ${ }^{1}$, \\ Do Ngoc Dai ${ }^{4}$, Le Thi Huong ${ }^{5}$, Thieu Anh Tai ${ }^{6}$ and William N. Setzer ${ }^{2,7, * \mathbb{D}}$ \\ 1 Center for Advanced Chemistry, Institute of Research and Development, Duy Tan University,03 Quang \\ Trung, Da Nang 50000, Vietnam; huyhung2602@gmail.com (N.H.H.); \\ hongchuong1991@gmail.com (N.T.H.C.) \\ 2 Aromatic Plant Research Center, 230 N 1200 E, Suite 102, Lehi, UT 84043, USA; psatyal@aromaticplant.org \\ 3 Parasitology and Entomology Unit, Department of Medicine, Duy Tan University, 03 Quang Trung, \\ Da Nang 50000, Vietnam \\ 4 Faculty of Agriculture, Forestry and Fishery, Nghe An Economics University, Vinh City 43000, Nghe An \\ Province, Vietnam; daidn23@gmail.com \\ 5 School of Natural Science Education, Vinh University, 182 Le Duan, Vinh City 43000, Nghệ An Province, \\ Vietnam; lehuong223@gmail.com \\ 6 Department of Pharmacy, Duy Tan University, 03-Quang Trung, Da Nang 50000, Vietnam; \\ anhtai0808qn@gmail.com \\ 7 Department of Chemistry, University of Alabama in Huntsville, Huntsville, AL 35899, USA \\ * Correspondence: hieuhoviet@gmail.com (H.V.H.); wsetzer@chemistry.uah.edu (W.N.S.)
}

Received: 27 December 2018; Accepted: 31 January 2019; Published: 3 February 2019

\begin{abstract}
Mosquito-borne infections are a constant problem in Vietnam, and mosquito vector control is a primary approach to control these infections. Essential oils represent environmentally friendly alternatives to synthetic pesticides for mosquito control. The essential oils of two weedy species in Vietnam, Erechtites hieraciifolius and E. valerianifolius, have been obtained by hydrodistillation and analyzed by gas chromatography-mass spectrometry. The essential oils have been screened for mosquito larvicidal activity against Aedes albopictus, Ae. aegypti, and Culex quinquefasciatus. The essential oil from the aerial parts of E. hieraciifolius was rich in $\alpha$-pinene (14.5\%), limonene $(21.4 \%)$, and caryophyllene oxide $(15.1 \%)$, while E. valerianifolius essential oil was dominated by myrcene $(47.8 \%)$ and $\alpha$-pinene $(30.2 \%)$. Both essential oils showed good larvicidal activity against Ae. albopictus (24-h LC $\mathrm{LC}_{50} 10.5$ and $5.8 \mu \mathrm{g} / \mathrm{mL}$, respectively) and Ae. aegypti (24-h LC $\mathrm{LC}_{50} 10.6$ and $12.5 \mu \mathrm{g} / \mathrm{mL}$, respectively). The essential oil of E. valerianifolius also showed good activity against $C x$. quinquefasciatus larvae (24-h LC $50=40.7 \mu \mathrm{g} / \mathrm{mL})$. Thus, Erechtites essential oils may serve as low-cost vector control agents for mosquito-borne infections.
\end{abstract}

Keywords: Erechtites hieraciifolius; Erechtites valerianifolius; chemical composition; $\alpha$-pinene; limonene; myrcene; $\beta$-caryophyllene; caryophyllene oxide

\section{Introduction}

Aedes aegypti (L.) and Ae. albopictus (Skuse) (Diptera: Culicidae) are important vectors of arboviral infections, including yellow fever, dengue, Zika, and chikungunya [1-3]. Vietnam is classified as a hyperendemic dengue country, with all four dengue serotypes present throughout the year [4]. In the last half century, dengue fever epidemics have increased in frequency, corresponding to a median annual incidence of 232 cases per 100,000 people [4]. Furthermore, chikungunya is expected to become a major health threat in Vietnam in the near future [4,5]. 
Vector control is one of the primary approaches to reduce the spread of arboviral infections. However, current methods for controlling Aedes mosquitoes have been largely ineffective [6]. Botanical insecticides in general $[7,8]$ and essential oils in particular $[9,10]$ have emerged as promising, environmentally friendly alternatives to synthetic pesticides for mosquito control.

There are around 12 species of Erechtites (Asteraceae), and they are native to North America, West Indies, South America, New Zealand, and Australia [11]. Erechtites hieraciifolius (L.) Raf. ex DC. (syn. Erechtites hieracifolia (L.) Raf., Erechtites hieraciifolia (L.) Raf. ex DC.,) is native to North America, South America, and the West Indies, but it has been introduced to Europe, Hawaii, and Asia [12-16]. Erechtites valerianifolius (Wolf) DC. (syn. Erechtites valerianifolia (Link ex Wolf) Less. ex DC., Erechtites valerianaefolia (Wolf) DC.) is native to Central and South America, but this species has also has been introduced to Asia [13-15,17,18].

Erechtites hieraciifolius is used traditionally in Venezuela (a plant decoction is used as a bath to reduce fever) and in El Salvador (a decoction is used to treat coughs) [19]. In Bolivia, the Tacana people use an oil extract of E. hieraciifolius to treat wounds and pimples [20]. An ethanol extract of $E$. hieraciifolius showed in vitro antileishmanial activity against promastigotes of Leishmania (Leishmania) amazonensis Lainson \& Shaw and L. (Viannia) braziliensis Vianna [20]. In North America, E. hieraciifolius was previously used to treat hemorrhages, wounds, skin diseases, and as a topical treatment for poison ivy (Toxicodendron radicans (L.) Kuntze, Anacardiaceae) and poison sumac (T. vernix (L.) Kuntze) rash [21].

As part of our ongoing research on identifying the potential utility of invasive plant species in Vietnam, we have obtained the essential oils from E. hieraciifolius and E. valerianifolius and have examined their mosquito larvicidal activities. In order to assess the potential environmental impact of using Erechtites essential oils as a larvicidal control agent, we have carried out lethality assays on the non-target aquatic species. As far as we are aware, there have been no previous investigations on the larvicidal activities of Erechtites essential oils.

\section{Materials and Methods}

\subsection{Plant Material}

Aerial parts of E. valerianifolius were harvested from plants growing in Dong Giang district, Quang Nam Province $\left(15^{\circ} 58^{\prime} 9.8^{\prime \prime} \mathrm{N}, 107^{\circ} 55^{\prime} 4.7^{\prime \prime}\right.$ E; sample Quang Nam), Hoa Vang district, Da Nang city $\left(16^{\circ} 01^{\prime} 0.6^{\prime \prime} \mathrm{N}, 108^{\circ} 4^{\prime} 25.6^{\prime \prime} \mathrm{E}\right.$;), while aerial parts of $E$. hieraciifolius were harvested from plants growing in Hoa Vang district, Da Nang city $\left(16^{\circ} 2^{\prime} 22.0^{\prime \prime} \mathrm{N}, 108^{\circ} 3^{\prime} 33.0^{\prime \prime} \mathrm{E}\right)$, in April 2018. The plants were identified by Dr. Do Ngoc Dai, and voucher specimens (LTH127 and LTH128, respectively) have been deposited in the Pedagogical Institute of Science, Vinh University. Fresh plant materials (leaves, stems, and flowers) were kept at room temperature $\left(\approx 25^{\circ} \mathrm{C}\right)$, and $2 \mathrm{~kg}$ samples of each of the plant materials were shredded and hydrodistilled for $4 \mathrm{~h}$ using a Clevenger type apparatus.

\subsection{Gas Chromatographic-Mass Spectral Analysis}

Each of the Erechtites essential oils was analyzed by gas chromatography-mass spectrometry (GC-MS) using a Shimadzu GCMS-QP2010 Ultra operated in the electron impact (EI) mode (electron energy $=70 \mathrm{eV}$ ), scan range $=40-400$ atomic mass units, scan rate $=3.0 \mathrm{scans} / \mathrm{s}$, and GC-MS solution software. The GC column was a ZB-5 fused silica capillary column with a (5\% phenyl)-polymethylsiloxane stationary phase and a film thickness of $0.25 \mu \mathrm{m}$. The carrier gas was helium with a column head pressure of $552 \mathrm{kPa}$ and flow rate of $1.37 \mathrm{~mL} / \mathrm{min}$. The injector temperature was $250{ }^{\circ} \mathrm{C}$ and the ion source temperature was $200{ }^{\circ} \mathrm{C}$. The GC oven temperature program was programmed to have an initial temperature of $50{ }^{\circ} \mathrm{C}$, and the temperature increased at a rate of $2{ }^{\circ} \mathrm{C} / \mathrm{min}$ to $260{ }^{\circ} \mathrm{C}$. A $5 \% \mathrm{w} / v$ solution of the sample in $\mathrm{CH}_{2} \mathrm{Cl}_{2}$ was prepared, and $0.1 \mu \mathrm{L}$ was injected with a splitting mode (30:1). Identification of the oil components was based on their retention indices determined by reference to a homologous series of $n$-alkanes, and by comparison of their mass 
spectral fragmentation patterns with those reported in the literature [22], and stored in our in-house Sat-Set library [23].

\subsection{Mosquito Larvicidal Assay}

Laboratory-reared larvae of Ae. aegypti and Ae. albopictus were collected from a mosquito colony maintained at the Laboratory of Parasitology and Entomology of Duy Tan University, Da Nang Vietnam. Wild larvae of Ae. albopictus and Culex quinquefasciatus (Say) were collected from Hoa Khanh Nam district $\left(16^{\circ} 3^{\prime} 14.9^{\prime \prime} \mathrm{N}, 108^{\circ} 9^{\prime} 31.2^{\prime \prime} \mathrm{E}\right)$. For the assay, aliquots of the aerial parts (leaves and stems) and essential oils of E. hieraciifolius and E. valerianifolius (Quang Nam stems \& leaves) dissolved in dimethylsulfoxide (DMSO) (1\% stock solution of essential oil in DMSO) were placed in $500 \mathrm{~mL}$ beakers and added to water that contained 25 larvae (fourth instar). With each experiment, a set of controls using DMSO was also run for comparison. Mortality was recorded after $24 \mathrm{~h}$ and again after $48 \mathrm{~h}$ of exposure, during which no nutritional supplement was added. The experiments were carried out at $25 \pm 2{ }^{\circ} \mathrm{C}$. Each test was conducted with four replicates with six concentrations $(100,80,50,25,12.5$, and $5 \mu \mathrm{g} / \mathrm{mL})$. Permethrin was used as a positive control.

\subsection{Non-Target Lethality Assays}

For the assay against Daphnia magna Straus (Cladocera: Daphniiidae), aliquots of the essential oil of E. hieraciifolius and E. valerianifolius (Quang Nam stems and leaves), dissolved in DMSO (1\% stock solution), were placed in $250 \mathrm{~mL}$ beakers and added to water that contained 20 larvae (fourth instar). Mortality was recorded after $24 \mathrm{~h}$ and $48 \mathrm{~h}$ of exposure, during which no nutritional supplement was added. The experiments were carried out at $25 \pm 2{ }^{\circ} \mathrm{C}$. Each test was conducted with four replicates with five concentrations $(12,6,3,1.5$, and $0.75 \mu \mathrm{g} / \mathrm{mL})$. The assay against Chiromonus tentans Fabricius (Diptera: Chironomidae) larvae was carried out as above using four replicates with five concentrations $(100,50,25,12.5$, and $6 \mu \mathrm{g} / \mathrm{mL})$. For the assay against Danio rerio Hamilton (Cypriniformes: Cyprinidae), young, immature fish around $2-3 \mathrm{~cm}$ in size were selected for the experiment. Twenty fish were separated in $2.5 \mathrm{~L}$ plastic containers with $1.0 \mathrm{~L}$ of tap water, with a temperature of $25 \pm 2{ }^{\circ} \mathrm{C}$ and external relative humidity of $85 \%$. For each dose $(100,50,25,12.5$, and $6 \mu \mathrm{g} / \mathrm{mL}$ ), four repetitions of the experiment were performed. The mortality of organism non-target was calculated following an exposure period of $24 \mathrm{~h}$. With each experiment, a set of controls using DMSO was also run for comparison.

\subsection{Data Analysis}

The mortalities were recorded $24 \mathrm{~h}$ and $48 \mathrm{~h}$ after treatment. The data obtained were subjected to log-probit analysis [24] to obtain LC $_{50}$ values, LC $_{90}$ values, 95\% confidence limits, and chi square values using Minitab ${ }^{\circledR} 18$ (Minitab Inc., State College, PA, USA). For comparison, LC $_{50}$ values were also determined using the Reed-Muench method [25].

\section{Results and Discussion}

The essential oils from the aerial parts of E. valerianifolius and E. hieraciifolius were obtained in $1.53 \%$ and $1.47 \%$ yields, respectively.

\subsection{Essential Oil Compositions}

The chemical compositions of the essential oil of E. hieraciifolius and E. valerianifolius are presented in Tables 1 and 2, respectively. The essential oil from the aerial parts (leaves and stems) of $E$. hieraciifolius was rich in the monoterpene hydrocarbons $\alpha$-pinene $(14.5 \%)$ and limonene $(21.4 \%)$, as well as the oxygenated sesquiterpenoid caryophyllene oxide (15.1\%). The floral essential oil of $E$. hieraciifolius was also rich in $\alpha$-pinene $(11.8 \%)$ and limonene $(29.8 \%)$, but $\beta$-caryophyllene $(22.1 \%)$ was the dominant sesquiterpene. 
Table 1. Chemical compositions of Erechtites hieraciifolius essential oils from Vietnam.

\begin{tabular}{|c|c|c|c|}
\hline \multirow{2}{*}{ RI } & \multirow{2}{*}{ Compound } & \multicolumn{2}{|c|}{ Area \% } \\
\hline & & Leaves \& Stems & Flowers \\
\hline 921 & Tricyclene & - & $\operatorname{tr}$ \\
\hline 924 & $\alpha$-Thujene & - & $\operatorname{tr}$ \\
\hline 932 & $\alpha$-Pinene & 14.5 & 11.8 \\
\hline 948 & Camphene & - & 0.1 \\
\hline 971 & Sabinene & 0.6 & 0.7 \\
\hline 976 & $\beta$-Pinene & 0.4 & 0.4 \\
\hline 988 & Myrcene & 2.7 & 4.4 \\
\hline 1006 & $\alpha$-Phellandrene & - & 0.3 \\
\hline 1016 & $\alpha$-Terpinene & - & $\operatorname{tr}$ \\
\hline 1024 & $p$-Cymene & 0.4 & 0.1 \\
\hline 1028 & Limonene & 21.4 & 29.8 \\
\hline 1031 & $\beta$-Phellandrene & - & 0.5 \\
\hline 1034 & (Z)- $\beta$-Ocimene & - & 1.2 \\
\hline 1044 & (E)- $\beta$-Ocimene & - & 2.3 \\
\hline 1057 & $\gamma$-Terpinene & - & 0.1 \\
\hline 1084 & Terpinolene & - & 0.1 \\
\hline 1108 & Unidentified & 0.8 & - \\
\hline 1120 & trans- $p$-Mentha-2,8-dien-1-ol & 0.8 & - \\
\hline 1124 & Cycloctanone & 0.6 & - \\
\hline 1125 & $\alpha$-Campholenal & 0.6 & - \\
\hline 1127 & allo-Ocimene & - & $\operatorname{tr}$ \\
\hline 1135 & cis-p-Mentha-2,8-dien-1-ol & 0.9 & - \\
\hline 1140 & trans-Pinocarveol & 0.7 & - \\
\hline 1140 & cis-Verbenol & 0.3 & - \\
\hline 1144 & trans-Verbenol & 3.5 & - \\
\hline 1179 & Terpinen-4-ol & 0.4 & - \\
\hline 1185 & Cryptone & 1.4 & - \\
\hline 1194 & Myrtenol & 0.8 & - \\
\hline 1197 & Dodecane & - & 0.1 \\
\hline 1198 & cis-Piperitol & 0.8 & - \\
\hline 1205 & Verbenone & 1.4 & - \\
\hline 1209 & Unidentified & 0.5 & - \\
\hline 1214 & Unidentified & 1.1 & - \\
\hline 1217 & trans-Carveol & 3.5 & - \\
\hline 1225 & Unidentified & 0.7 & - \\
\hline 1230 & cis-Carveol & 1.1 & - \\
\hline 1242 & Carvone & 2.0 & - \\
\hline 1270 & Unidentified & 0.8 & - \\
\hline 1284 & Bornyl acetate & - & 0.2 \\
\hline 1287 & Limonene dioxide & 0.9 & - \\
\hline 1297 & Tridecane & - & 0.2 \\
\hline 1309 & Unidentified & 2.2 & - \\
\hline 1317 & 3-Hydroxycineole & 0.4 & - \\
\hline 1343 & Limonene-1,2-diol & 4.7 & - \\
\hline 1345 & $\alpha$-Cubebene & - & 0.1 \\
\hline 1357 & Neryl acetate & - & 0.1 \\
\hline 1367 & Cyclosativene & - & 0.1 \\
\hline 1374 & $\alpha$-Copaene & 0.6 & 1.9 \\
\hline 1378 & trans-p-Menth-6-en-2,8-diol & 4.1 & - \\
\hline 1386 & $\beta$-Cubebene & - & 0.7 \\
\hline 1387 & $\beta$-Elemene & 0.6 & 3.5 \\
\hline 1397 & Tetradecane & - & 0.2 \\
\hline 1402 & $\alpha$-Gurjunene & - & 1.1 \\
\hline 1419 & $\beta$-Caryophyllene & 3.0 & 22.1 \\
\hline 1450 & $(E)$ - $\beta$-Farnesene & - & 2.0 \\
\hline 1454 & $\alpha$-Humulene & 0.5 & 1.8 \\
\hline 1470 & trans-Cadina-1(6),4-diene & - & 0.1 \\
\hline 1472 & $\gamma$-Gurjunene & - & 0.2 \\
\hline
\end{tabular}


Table 1. Cont.

\begin{tabular}{|c|c|c|c|}
\hline \multirow{2}{*}{ RI } & \multirow{2}{*}{ Compound } & \multicolumn{2}{|c|}{ Area \% } \\
\hline & & Leaves \& Stems & Flowers \\
\hline 1473 & $\gamma$-Muurolene & - & 0.1 \\
\hline 1480 & Germacrene D & - & 2.6 \\
\hline 1482 & $(Z, Z)-\alpha$-Farnesene & - & 0.7 \\
\hline 1486 & Valencene & - & 0.7 \\
\hline 1488 & Viridiflorene & - & 0.7 \\
\hline 1490 & trans-Muurola-4(14),5-diene & - & 0.3 \\
\hline 1494 & epi-Cubebol & - & 0.5 \\
\hline 1496 & $\alpha$-Muurolene & - & 1.2 \\
\hline 1501 & $(E, E)-\alpha$-Farnesene & - & 0.1 \\
\hline 1514 & Cubebol & - & 0.2 \\
\hline 1516 & $\delta$-Cadinene & - & 1.4 \\
\hline 1549 & Isocaryphyllene oxide & 1.2 & - \\
\hline 1559 & (E)-Nerolidol & - & 0.3 \\
\hline 1582 & Caryophyllene oxide & 15.1 & 1.6 \\
\hline 1607 & Humulene epoxide II & 0.9 & - \\
\hline 1622 & Cyperotundone A & - & 0.1 \\
\hline 1627 & 1-epi-Cubenol & - & 0.2 \\
\hline 1637 & Caryophylla-4(12),8(13)-dien-5 $\beta$-ol & 0.6 & 0.1 \\
\hline 1641 & $\tau$-Cadinol & - & 0.4 \\
\hline 1643 & $\tau$-Muurolol & - & 0.2 \\
\hline 1644 & Cubenol & 0.5 & - \\
\hline 1646 & $\alpha$-Muurolol (Torreyol) & - & 0.1 \\
\hline 1654 & $\alpha$-Cadinol & 0.7 & 0.3 \\
\hline 1658 & Selin-11-en- $4 \alpha$-ol & - & 0.1 \\
\hline 1667 & 14-Hydroxy-9-epi-(E)-caryophyllene & 1.4 & - \\
\hline 1700 & Heptadecane & - & 0.2 \\
\hline 1831 & Neophytadiene & - & 0.3 \\
\hline 1900 & Nonadecane & - & 0.2 \\
\hline \multirow[t]{7}{*}{2103} & (E)-Phytol & - & 0.4 \\
\hline & Monoterpene hydrocarbons & 40.0 & 51.7 \\
\hline & Oxygenated monoterpenoids & 28.2 & 0.3 \\
\hline & Sesquiterpene hydrocarbons & 4.7 & 41.3 \\
\hline & Oxygenated sesquiterpenoids & 19.2 & 4.1 \\
\hline & Others & 0.6 & 1.5 \\
\hline & Total Identified & 92.7 & 99.0 \\
\hline
\end{tabular}

Table 2. Chemical compositions of Erechtites valerianifolius essential oils from Vietnam.

\begin{tabular}{ccccc}
\hline RI & Compound & $\begin{array}{c}\text { Quang Nam } \\
\text { Leaves \& stems }\end{array}$ & $\begin{array}{c}\text { Quang Nam } \\
\text { Flowers }\end{array}$ & $\begin{array}{c}\text { Da Nang } \\
\text { Flowers }\end{array}$ \\
\hline 922 & Tricyclene & $\operatorname{tr}$ & $\operatorname{tr}$ & $\operatorname{tr}$ \\
924 & $\alpha$-Thujene & $\operatorname{tr}$ & 0.1 & $\operatorname{tr}$ \\
933 & $\alpha$-Pinene & 30.2 & 32.5 & 30.6 \\
949 & Camphene & 0.1 & 0.1 & 0.1 \\
952 & Thuja-2,4(10)-diene & $\operatorname{tr}$ & $\operatorname{tr}$ & $\operatorname{tr}$ \\
971 & Sabinene & 0.7 & 0.4 & 0.9 \\
977 & $\beta$-Pinene & 0.3 & 57.0 & 0.3 \\
990 & Myrcene & 47.8 & $\operatorname{tr}$ & 60.6 \\
1006 & $\alpha$-Phellandrene & 0.3 & $\operatorname{tr}$ & $\operatorname{tr}$ \\
1016 & $\alpha$-Terpinene & $\operatorname{tr}$ & $\operatorname{tr}$ & $\operatorname{tr}$ \\
1024 & $p$-Cymene & 0.1 & 2.5 & 1.5 \\
1028 & Limonene & 1.4 & 0.2 & 0.2 \\
1030 & $\beta$-Phellandrene & 0.1 & & \\
\hline
\end{tabular}


Table 2. Cont.

\begin{tabular}{|c|c|c|c|c|}
\hline RI & Compound & $\begin{array}{c}\text { Quang Nam } \\
\text { Leaves \& stems }\end{array}$ & $\begin{array}{l}\text { Quang Nam } \\
\text { Flowers }\end{array}$ & $\begin{array}{c}\text { Da Nang } \\
\text { Flowers }\end{array}$ \\
\hline 1034 & (Z)- $\beta$-Ocimene & 0.3 & 0.1 & $\operatorname{tr}$ \\
\hline 1044 & (E)- $\beta$-Ocimene & 1.4 & 0.4 & 0.2 \\
\hline 1057 & $\gamma$-Terpinene & 0.1 & 0.1 & 0.1 \\
\hline 1084 & Terpinolene & $\operatorname{tr}$ & 0.1 & 0.1 \\
\hline 1100 & Undecane & - & $\operatorname{tr}$ & $\operatorname{tr}$ \\
\hline 1101 & Perillene & 0.1 & $\operatorname{tr}$ & $\operatorname{tr}$ \\
\hline 1102 & Linalool & $\operatorname{tr}$ & $\operatorname{tr}$ & $\operatorname{tr}$ \\
\hline 1112 & (E)-4,8-Dimethylnona-1,3,7-triene & $\operatorname{tr}$ & $\operatorname{tr}$ & $\operatorname{tr}$ \\
\hline 1128 & $\alpha$-Campholenal & 0.1 & - & - \\
\hline 1146 & trans-Verbenol & - & $\operatorname{tr}$ & $\operatorname{tr}$ \\
\hline 1181 & Terpinen-4-ol & 0.1 & $\operatorname{tr}$ & $\operatorname{tr}$ \\
\hline 1229 & Thymol methyl ether & $\operatorname{tr}$ & - & - \\
\hline 1333 & $\delta$-Elemene & 0.1 & 0.1 & 0.1 \\
\hline 1374 & $\alpha$-Copaene & 0.1 & $\operatorname{tr}$ & $\operatorname{tr}$ \\
\hline 1380 & cis- $\beta$-Elemene & 0.1 & $\operatorname{tr}$ & $\operatorname{tr}$ \\
\hline 1382 & $\beta$-Bourbonene & $\operatorname{tr}$ & $\operatorname{tr}$ & $\operatorname{tr}$ \\
\hline 1386 & $\beta$-Cubebene & - & $\operatorname{tr}$ & 0.3 \\
\hline 1387 & $\beta$-Elemene & 2.4 & 0.2 & 0.1 \\
\hline 1400 & Methyl eugenol & $\operatorname{tr}$ & - & - \\
\hline 1401 & $\alpha$-Gurjunene & 0.1 & - & - \\
\hline 1411 & Dimethoxy- $p$-cymene & 0.2 & - & - \\
\hline 1418 & $\beta$-Caryophyllene & 5.4 & 2.7 & 2.2 \\
\hline 1427 & $\gamma$-Elemene & 0.1 & $\operatorname{tr}$ & $\operatorname{tr}$ \\
\hline 1428 & $\beta$-Copaene & 0.1 & $\operatorname{tr}$ & $\operatorname{tr}$ \\
\hline 1450 & (E)- $\beta$-Farnesene & 0.2 & $\operatorname{tr}$ & $\operatorname{tr}$ \\
\hline 1454 & $\alpha$-Humulene & 0.7 & 0.3 & 0.3 \\
\hline 1471 & $\gamma$-Selinene & 0.2 & - & - \\
\hline 1473 & $\gamma$-Muurolene & 0.1 & $\operatorname{tr}$ & $\operatorname{tr}$ \\
\hline 1480 & Germacrene D & 3.3 & 1.8 & 1.8 \\
\hline 1486 & Viridiflorene & 0.3 & - & - \\
\hline 1488 & $\beta$-Selinene & 0.2 & $\operatorname{tr}$ & $\operatorname{tr}$ \\
\hline 1491 & trans-Muurola-4(14),5-diene & 0.1 & $\operatorname{tr}$ & $\operatorname{tr}$ \\
\hline 1494 & $\alpha$-Selinene & 0.4 & - & - \\
\hline 1494 & Bicyclogermacrene & - & 0.1 & 0.2 \\
\hline 1496 & $\alpha$-Muurolene & 0.1 & 0.1 & $\operatorname{tr}$ \\
\hline 1501 & $(E, E)-\alpha$-Farnesene & 0.7 & $\operatorname{tr}$ & 0.1 \\
\hline 1511 & $\gamma$-Cadinene & $\operatorname{tr}$ & $\operatorname{tr}$ & $\operatorname{tr}$ \\
\hline 1516 & $\delta$-Cadinene & 0.2 & 0.1 & 0.1 \\
\hline 1558 & Germacrene B & 0.1 & $\operatorname{tr}$ & $\operatorname{tr}$ \\
\hline 1576 & Spathulenol & 0.1 & $\operatorname{tr}$ & $\operatorname{tr}$ \\
\hline 1582 & Caryophyllene oxide & 0.7 & 0.1 & 0.1 \\
\hline 1609 & Humulene epoxide II & 0.1 & - & - \\
\hline 1622 & Cyperotundone A & 0.1 & - & - \\
\hline 1627 & iso-Spathulenol & tr & - & - \\
\hline 1642 & $\tau$-Cadinol & 0.1 & $\operatorname{tr}$ & $\operatorname{tr}$ \\
\hline 1643 & $\tau$-Muurolol & 0.1 & $\operatorname{tr}$ & $\operatorname{tr}$ \\
\hline 1655 & $\alpha$-Cadinol & 0.1 & $\operatorname{tr}$ & $\operatorname{tr}$ \\
\hline 1659 & Selin-11-en- $4 \alpha$-ol & 0.1 & - & - \\
\hline 1684 & Germacra-4(15),5,10(14)-trien- $1 \alpha$-ol & - & - & $\operatorname{tr}$ \\
\hline 1700 & Heptadecane & 0.1 & 0.1 & 0.1 \\
\hline 1832 & Neophytadiene & 0.2 & - & $\operatorname{tr}$ \\
\hline 1900 & Nonadecane & - & $\operatorname{tr}$ & 0.1 \\
\hline 1944 & $\alpha$-Springene & 0.1 & 0.1 & 0.1 \\
\hline \multirow[t]{7}{*}{2100} & Heneicosane & - & $\operatorname{tr}$ & $\operatorname{tr}$ \\
\hline & Monoterpene hydrocarbons & 82.9 & 94.3 & 94.6 \\
\hline & Oxygenated monoterpenoids & 0.3 & $\operatorname{tr}$ & $\operatorname{tr}$ \\
\hline & Sesquiterpene hydrocarbons & 14.9 & 5.4 & 5.1 \\
\hline & Oxygenated sesquiterpenoids & 1.3 & 0.1 & 0.1 \\
\hline & Others & 0.4 & 0.1 & 0.2 \\
\hline & Total Identified & 99.9 & 100.0 & 100.0 \\
\hline
\end{tabular}


The essential oil from the aerial parts (stems and leaves) of E. valerianifolius was dominated by the monoterpene hydrocarbons myrcene $(47.8 \%)$ and $\alpha$-pinene $(30.2 \%)$, with a lesser quantity of the sesquiterpene $\beta$-caryophyllene (5.4\%) (Table 2 ). The floral essential oils of E. valerianifolius were also rich in myrcene (57.0 and $60.6 \%$ ) and $\alpha$-pinene (32.5 and $30.6 \%$ ).

Erechtites hieraciifolius and E. valerianifolius essential oils from other geographical locations have shown wide variations in chemical composition (Table 3). Thus, $\alpha$-phellandrene (41.3\%) and $p$-cymene (22.2\%) dominated the essential oil of E. hieraciifolius from Pacoti-Ceara, Brazil [26], while these compounds were only minor components in the sample from Vietnam. Likewise, dillapiole (33.8\%) was the major component in E. hieraciifolius from Parana State, Brazil [27]; this compound was not observed in the essential oils from Vietnam. The essential oil compositions of E. valerianifolius from Vietnam were qualitatively similar to those reported by do Amaral and co-workers from southern Brazil [27], but with major quantitative differences.

It is not clear why there is so much variation in the essential oils of Erechtites species. The phytochemical variations may be due to genetic variation. For example, the Missouri Botanical Garden [28] lists six varieties of $H$. hieraciifolius native to the Americas: var. cacalioides (Fisch. Ex Spreng.) Griseb (West Indies, Central and South America), var. carduifolius (Cass.) Griseb (West Indies), var. hieraciifolius (North America and West Indies), var. intermedia Fernald (North America), var. megalocarpus (Fernald) Cronquist (North America), and var. praealtus (Raf.) Fernald (North America). In addition, climatic and edaphic factors, maturity, and phenology can also be responsible for phytochemical variations, particularly in wide-ranging species. For example, several chemotypes of Artemisia absinthium L. (Asteraceae) are known, based largely on geographical location [29]. The essential oil of Peperomia pelucida (L.) Kunth (Piperaceae) also shows wide variation depending on the geographical source of material [30].

Table 3. Major chemical components ( $>5 \%)$ of Erechtites essential oils.

\begin{tabular}{|c|c|c|c|}
\hline Erechtites Species & Geographical Location & Major Components & Ref. \\
\hline E. hieraciifolius & Pacoti-Ceara, Brazil & $\begin{array}{c}\alpha \text {-phellandrene }(41.3 \%), \\
p \text {-cymene }(22.2 \%), \\
\beta \text {-caryophyllene }(7.4 \%), \\
\text { camphor }(5.4 \%)\end{array}$ & [26] \\
\hline E. hieraciifolius & $\begin{array}{c}\text { Chimoré area, Chapare } \\
\text { Province, Bolivia }\end{array}$ & $\begin{array}{c}\alpha \text {-pinene }(48.0 \%), \\
(E) \text { - } \beta \text {-ocimene }(13.9 \%), \\
\text { myrcene }(13.7 \%)\end{array}$ & [31] \\
\hline E. hieraciifolius & $\begin{array}{c}\text { "Private Reservation of } \\
\text { Natural Heritage", Parana } \\
\text { State, Brazil }\end{array}$ & $\begin{array}{c}\text { dillapiole }(33.8 \%), \\
\alpha \text {-pinene }(33.0 \%), \\
\beta \text {-pinene }(14.7 \%), \\
\text { limonene }(9.7 \%)\end{array}$ & [27] \\
\hline E. valerianifolius & Mérida, Venezuela & $\begin{array}{c}\text { limonene }(56.7 \%), \\
\text { myrcene }(12.7 \%), \\
(E) \text { - } \beta \text {-farnesene }(10.2 \%), \\
\alpha \text {-phellandrene }(8.7 \%)\end{array}$ & [32] \\
\hline E. valerianifolius & $\begin{array}{c}\text { "Private Reservation of } \\
\text { Natural Heritage", Parana } \\
\text { State, Brazil }\end{array}$ & $\begin{array}{l}\alpha \text {-pinene }(25.8 \%), \\
\text { sabinene }(17.0 \%), \\
\text { myrcene }(16.7 \%), \\
\beta \text {-pinene }(13.3 \%), \\
\text { limonene }(12.6 \%)\end{array}$ & [27] \\
\hline
\end{tabular}

\subsection{Mosquito Larvicidal Activities}

The essential oils from the aerial parts of E. hieraciifolius and E. valerianifolius collected from Vietnam were screened for mosquito larvicidal activity (Tables 4 and 5). Larvicidal activity of permethrin (positive control) is shown in Table 6. 
Table 4. Mosquito larvicidal activity of Erechtites hieraciifolius aerial parts (leaves and stems) essential oil.

\begin{tabular}{|c|c|c|c|c|c|c|}
\hline Mosquito Species & $\begin{array}{l}\text { Treatment } \\
\text { Time }\end{array}$ & $\begin{array}{c}\mathrm{LC}_{50, \mu \mathrm{g} / \mathrm{Ml}{ }^{\mathrm{a}}} \\
\text { (Fiducial } \\
\text { Limits) }\end{array}$ & $\begin{array}{c}\mathrm{LC}_{90,} \mu \mathrm{g} / \mathrm{Ml}{ }^{\mathrm{a}} \\
\text { (Fiducial } \\
\text { Limits) }\end{array}$ & $\begin{array}{l}\text { Regression } \\
\text { Equation }\end{array}$ & $x^{2}$ & $p$ \\
\hline Ae. Albopictus ${ }^{\mathrm{b}}$ & $24 \mathrm{~h}$ & $\begin{array}{c}10.47 \\
(9.12-11.70) \\
10.06 \pm 0.92 \\
\end{array}$ & $\begin{array}{c}21.11 \\
(19.28-23.59)\end{array}$ & $\begin{array}{c}y=-1.764+ \\
0.1443 x\end{array}$ & 17.6 & $<0.001$ \\
\hline Ae. Albopictus ${ }^{\mathrm{b}}$ & $48 \mathrm{~h}$ & $\begin{array}{c}5.49 \\
(1.99-7.87) \\
6.50 \pm 2.38 \\
\end{array}$ & $\begin{array}{c}18.64 \\
(15.95-22.92)\end{array}$ & $\begin{array}{c}y=-0.177+ \\
0.0782 x\end{array}$ & 12.68 & 0.002 \\
\hline Ae. Aegypti ${ }^{\mathrm{b}}$ & $24 \mathrm{~h}$ & $\begin{array}{c}10.58 \\
(9.42-11.68) \\
10.43 \pm 1.93 \\
\end{array}$ & $\begin{array}{c}19.47 \\
(17.82-21.76)\end{array}$ & $\begin{array}{c}y=-2.078+ \\
0.172 x\end{array}$ & 14.34 & 0.001 \\
\hline Ae. Aegypti ${ }^{\mathrm{b}}$ & $48 \mathrm{~h}$ & $\begin{array}{c}8.83 \\
(7.76-9.79) \\
8.65 \pm 1.56\end{array}$ & $\begin{array}{c}16.27 \\
(14.89-18.21)\end{array}$ & $\begin{array}{c}y=-2.073+ \\
0.206 x\end{array}$ & 35.49 & $<0.001$ \\
\hline
\end{tabular}

a There was no mortality in the dimethylsulfoxide (DMSO) controls; $\mathrm{LC}_{50}$ values in italics are from Reed-Muench analysis. ${ }^{\mathrm{b}}$ Laboratory-reared mosquito larvae.

Table 5. Mosquito larvicidal activity of Erechtites valerianifolius aerial parts (leaves and stems) essential oil.

\begin{tabular}{|c|c|c|c|c|c|c|}
\hline Mosquito Species & $\begin{array}{c}\text { Treatment } \\
\text { Time }\end{array}$ & $\begin{array}{c}\mathrm{LC}_{50}, \mu \mathrm{g} / \mathrm{Ml}^{\mathrm{a}} \\
\text { (Fiducial } \\
\text { Limits) }\end{array}$ & $\begin{array}{c}\mathrm{LC}_{90, \mu \mathrm{g} / \mathrm{Ml}{ }^{\mathrm{a}}} \\
\text { (Fiducial } \\
\text { Limits) }\end{array}$ & $\begin{array}{c}\text { Regression } \\
\text { Equation }\end{array}$ & $x^{2}$ & $p$ \\
\hline Ae. Albopictus ${ }^{\mathrm{b}}$ & $24 \mathrm{~h}$ & $\begin{array}{c}6.07 \\
(5.44-6.73) \\
6.38 \pm 0.72\end{array}$ & $\begin{array}{c}11.10 \\
(10.11-12.42)\end{array}$ & $\begin{array}{c}y=-2.110+ \\
0.306 x\end{array}$ & 1.02 & 0.599 \\
\hline Ae. Albopictus ${ }^{\mathrm{b}}$ & $48 \mathrm{~h}$ & $\begin{array}{c}4.65 \\
(4.11-5.25) \\
5.32 \pm 1.11\end{array}$ & $\begin{array}{c}9.01 \\
(7.96-10.67)\end{array}$ & $\begin{array}{c}y=-1.892+ \\
0.352 x\end{array}$ & 2.26 & 0.323 \\
\hline Ae. Albopictus ${ }^{\mathrm{c}}$ & $24 \mathrm{~h}$ & $\begin{array}{c}38.01 \\
(33.56-43.39) \\
40.71 \pm 8.44\end{array}$ & $\begin{array}{c}75.84 \\
(65.43-94.11)\end{array}$ & $\begin{array}{c}y=-1.796+ \\
0.041 x\end{array}$ & 5.83 & 0.016 \\
\hline Ae. Albopictus ${ }^{c}$ & $48 \mathrm{~h}$ & $\begin{array}{c}38.57 \\
(34.47-43.73) \\
35.59 \pm 6.58\end{array}$ & $\begin{array}{c}67.80 \\
(59.41-81.64)\end{array}$ & $\begin{array}{c}y=-1.691+ \\
0.044 x\end{array}$ & 5.36 & 0.021 \\
\hline Ae. Aegypti ${ }^{\mathrm{b}}$ & $24 \mathrm{~h}$ & $\begin{array}{c}12.56 \\
(11.21-13.84) \\
12.64 \pm 2.25\end{array}$ & $\begin{array}{c}23.72 \\
(21.78-26.34)\end{array}$ & $\begin{array}{c}y=-1.981+ \\
0.137 x\end{array}$ & 7.69 & 0.006 \\
\hline Ae. Aegypti ${ }^{\mathrm{b}}$ & $48 \mathrm{~h}$ & $\begin{array}{c}9.60 \\
(7.97-11.01) \\
9.40 \pm 1.55\end{array}$ & $\begin{array}{c}22.22 \\
(20.15-25.07)\end{array}$ & $\begin{array}{c}y=-1.422+ \\
0.122 x\end{array}$ & 22.53 & $<0.001$ \\
\hline Cx. Quinquefasciatus ${ }^{c}$ & $24 \mathrm{~h}$ & $\begin{array}{c}40.06 \\
(37.08-42.64) \\
40.00 \pm 4.92\end{array}$ & $\begin{array}{c}55.19 \\
(51.92-59.82)\end{array}$ & $\begin{array}{c}y=-4.316+ \\
0.101 x\end{array}$ & $5 \times 10^{-7}$ & 0.999 \\
\hline Cx. Quinquefasciatus ${ }^{c}$ & $48 \mathrm{~h}$ & $\begin{array}{c}39.48 \\
(36.73-42.23) \\
37.53 \pm 5.26\end{array}$ & $\begin{array}{c}53.18 \\
(49.70-58.00)\end{array}$ & $\begin{array}{c}y=-3.697+ \\
0.094 x\end{array}$ & $\begin{array}{l}1.2 \times \\
10^{-6}\end{array}$ & 0.999 \\
\hline
\end{tabular}

a There was no mortality in the DMSO controls; LC $_{50}$ values in italics are from Reed-Muench analysis. $b$

Laboratory-reared mosquito larvae. ${ }^{\mathrm{c}}$ Wild mosquito larvae. 
Table 6. Mosquito larvicidal activity of permethrin (positive control).

\begin{tabular}{|c|c|c|c|c|c|c|}
\hline Mosquito Species & $\begin{array}{c}\text { Treatment } \\
\text { Time }\end{array}$ & $\begin{array}{c}\mathrm{LC}_{50}, \mu \mathrm{g} / \mathrm{Ml} \mathrm{a}^{\mathrm{a}} \\
\text { (Fiducial Limits) }\end{array}$ & $\begin{array}{c}\mathrm{LC}_{90}, \mu \mathrm{g} / \mathrm{mL}^{\mathrm{a}} \\
\text { (Fiducial Limits) }\end{array}$ & $\begin{array}{c}\text { Regression } \\
\text { Equation }\end{array}$ & $x^{2}$ & $p$ \\
\hline Ae. Albopictus ${ }^{\mathrm{b}}$ & $24 \mathrm{~h}$ & $\begin{array}{c}0.0023 \\
(0.0021-0.0026) \\
0.0022 \pm 0.0003\end{array}$ & $\begin{array}{c}0.0042 \\
(0.0038-0.0049)\end{array}$ & $\begin{array}{c}y=-1.628+ \\
686.9 x\end{array}$ & 4.73 & 0.030 \\
\hline $\begin{array}{c}\text { Cx. } \\
\text { Quinquefasciatus }{ }^{\text {b }}\end{array}$ & $24 \mathrm{~h}$ & $\begin{array}{c}0.0167 \\
(0.0152-0.0183) \\
0.0148 \pm 0.0011\end{array}$ & $\begin{array}{c}0.0294 \\
(0.0270-0.0326)\end{array}$ & $\begin{array}{c}y=-2.292+ \\
121.6 x\end{array}$ & 26.62 & $<0.001$ \\
\hline
\end{tabular}

a There was no mortality in the DMSO controls; $\mathrm{LC}_{50}$ values in italics are from Reed-Muench analysis. ${ }^{\mathrm{b}}$ Wild mosquito larvae.

The essential oils from the aerial parts of both E. hieraciifolius and E. valerianifolius showed excellent larvicidal activity against Ae. aegypti. The $24 \mathrm{~h} \mathrm{LC} 50$ values were 10.6 and $12.5 \mu \mathrm{g} / \mathrm{mL}$, respectively, which compare very favorably with other essential oils reported in the literature against this species [33-35]. Similarly, the larvicidal activities for the two Erechtites essential oils against Ae. albopictus were also very encouraging, with $24 \mathrm{~h} \mathrm{LC}_{50}$ values of 10.5 and $5.8 \mu \mathrm{g} / \mathrm{mL}$ for E. hieraciifolius and E. valerianifoliu, respectively. Notably, the laboratory-reared Ae. albopictus larvae were more susceptible, based on the $95 \%$ confidence limits, to E. valerianifolius essential oil than the larvae obtained from the wild $\left(24 \mathrm{~h} \mathrm{LC}{ }_{50}=42.1 \mu \mathrm{g} / \mathrm{mL}\right)$. Likewise, wild Culex quinquefasciatus showed less susceptibility than the laboratory-reared mosquitoes.

Mosquito larvicidal activities $\left(\mathrm{LC}_{50}\right)$ of essential oils against $C x$. quinquefasciatus have generally ranged between $25.6 \mu \mathrm{g} / \mathrm{mL}$ and $225 \mu \mathrm{g} / \mathrm{mL}$ [36,37]. Thus, the $C x$. quinquefasciatus larvicidal activity of E. valerianifolius $\left(\mathrm{LC}_{50}=40.65 \mu \mathrm{g} / \mathrm{mL}\right.$ ) was good compared to other essential oils.

The major components of E. hieraciifolius aerial parts essential oil were $\alpha$-pinene, limonene, and caryophyllene oxide. Both $\alpha$-pinene and limonene have shown good larvicidal activities against Ae. aegypti and Ae. albopictus (see Table 7). The $\mathrm{LC}_{50}$ values for (+)-limonene average 35.1 and 29.8 against Ae. aegypti and Ae. albopictus, respectively. Caryophyllene oxide, however, has not shown good larvicidal activity, with $\mathrm{LC}_{50}$ values $>100 \mu \mathrm{g} / \mathrm{mL}$ against all mosquito species reported (Table 7).

Table 7. Mosquito larvicidal activities $\left(24 \mathrm{~h} \mathrm{LC}_{50}\right)$ of essential oil components against various mosquito species.

\begin{tabular}{cccc}
\hline Compound & Mosquito Species & LC $_{\mathbf{5 0}}(\boldsymbol{\mu g} / \mathbf{m L})$ & Ref. \\
\hline$\beta$-caryophyllene & Aedes aegypti & 88.30 & {$[38]$} \\
$\beta$-caryophyllene & Aedes aegypti & 38.58 & {$[39]$} \\
$\beta$-caryophyllene & Aedes albopictus & 44.77 & {$[40]$} \\
$\beta$-caryophyllene & Aedes albopictus & 39.52 & {$[39]$} \\
$\beta$-caryophyllene & Anopheles subpictus & 41.66 & {$[40]$} \\
$\beta$-caryophyllene & Culex pipiens pallens & 93.65 & {$[38]$} \\
$\beta$-caryophyllene & Culex pipiens pallens & 47.79 & {$[39]$} \\
$\beta$-caryophyllene & Culex tritaeniorhynchus & 48.17 & {$[40]$} \\
$\beta$-caryophyllene & Ochlerotatus togoi & 97.90 & {$[38]$} \\
caryophyllene oxide & Aedes aegypti & 125 & {$[41]$} \\
caryophyllene oxide & Aedes aegypti & 113.00 & {$[39]$} \\
caryophyllene oxide & Aedes albopictus & 107.62 & {$[39]$} \\
caryophyllene oxide & Culex pipiens pallens & 126.28 & {$[39]$} \\
limonene & Aedes aegypti & 19.4 & {$[42]$} \\
limonene & Aedes aegypti & 18.1 & {$[43]$} \\
limonene & Aedes albopictus & 15.0 & {$[42]$} \\
limonene & Aedes albopictus & 32.7 & {$[43]$} \\
(+)-limonene & Aedes aegypti & 27 & {$[44]$} \\
(+)-limonene & Aedes aegypti & 24.47 & {$[38]$} \\
(+)-limonene & Aedes aegypti & 71.9 & {$[45]$} \\
\hline
\end{tabular}


Table 7. Cont.

\begin{tabular}{|c|c|c|c|}
\hline Compound & Mosquito Species & $\mathrm{LC}_{50}(\mu \mathrm{g} / \mathrm{mL})$ & Ref. \\
\hline$(+)$-limonene & Aedes aegypti & 37 & {$[41]$} \\
\hline$(+)$-limonene & Aedes aegypti & 15.31 & [39] \\
\hline$(+)$-limonene & Aedes albopictus & 35.99 & [46] \\
\hline$(+)$-limonene & Aedes albopictus & 41.2 & [45] \\
\hline$(+)$-limonene & Aedes albopictus & 10.77 & [39] \\
\hline$(+)$-limonene & Aedes albopictus & 19.15 & [47] \\
\hline (+)-limonene & Aedes albopictus & 41.75 & [48] \\
\hline$(+)$-limonene & Culex pipiens pallens & 13.26 & [38] \\
\hline (+)-limonene & Culex pipiens pallens & 10.76 & [39] \\
\hline$(+)$-limonene & Culex quinquefasciatus & 40 & [49] \\
\hline$(+)$-limonene & Ochlerotatus togoi & 19.20 & [38] \\
\hline (-)-limonene & Aedes aegypti & 30 & [44] \\
\hline (-)-limonene & Aedes albopictus & 34.89 & [46] \\
\hline (-)-limonene & Aedes albopictus & 15.01 & [47] \\
\hline myrcene & Aedes aegypti & 35.8 & [43] \\
\hline myrcene & Aedes aegypti & 27.9 & [42] \\
\hline myrcene & Aedes aegypti & 66.42 & [38] \\
\hline myrcene & Aedes aegypti & 39.51 & [39] \\
\hline myrcene & Aedes albopictus & 27.0 & [43] \\
\hline myrcene & Aedes albopictus & 23.5 & [42] \\
\hline myrcene & Aedes albopictus & 35.98 & [39] \\
\hline myrcene & Aedes albopictus & 37.76 & [47] \\
\hline myrcene & Culex pipiens pallens & 66.28 & [38] \\
\hline myrcene & Culex pipiens pallens & 41.31 & [39] \\
\hline myrcene & Culex quinquefasciatus & 167 & [49] \\
\hline myrcene & Ochlerotatus togoi & 64.76 & [38] \\
\hline$\alpha$-pinene & Aedes aegypti & 15.4 & [50] \\
\hline$\alpha$-pinene & Aedes aegypti & 79.1 & [43] \\
\hline$\alpha$-pinene & Aedes albopictus & 74.0 & [43] \\
\hline$\alpha$-pinene & Aedes albopictus & 34.09 & [40] \\
\hline$\alpha$-pinene & Anopheles subpictus & 32.09 & [40] \\
\hline$\alpha$-pinene & Culex quinquefasciatus & 95 & [49] \\
\hline$\alpha$-pinene & Culex tritaeniorhynchus & 36.75 & [40] \\
\hline$(+)$ - $\alpha$-pinene & Aedes aegypti & 50.92 & [38] \\
\hline$(+)$ - $\alpha$-pinene & Aedes aegypti & 51.28 & [39] \\
\hline$(+)$ - $\alpha$-pinene & Aedes albopictus & 68.68 & [46] \\
\hline$(+)-\alpha$-pinene & Aedes albopictus & 55.65 & [39] \\
\hline$(+)$ - $\alpha$-pinene & Culex pipiens molestus & 47 & [51] \\
\hline$(+)$ - $\alpha$-pinene & Culex pipiens pallens & 53.96 & [38] \\
\hline$(+)$ - $\alpha$-pinene & Culex pipiens pallens & 60.84 & [39] \\
\hline$(+)$ - $\alpha$-pinene & Ochlerotatus togoi & 47.25 & [38] \\
\hline$(-)$ - $\alpha$-pinene & Aedes aegypti & 64.80 & [38] \\
\hline$(-)$ - $\alpha$-pinene & Aedes aegypti & 39.98 & [39] \\
\hline$(-)-\alpha$-pinene & Aedes albopictus & 72.30 & [46] \\
\hline$(-)-\alpha$-pinene & Aedes albopictus & 28.61 & [39] \\
\hline$(-)-\alpha$-pinene & Culex pipiens molestus & 49 & [51] \\
\hline$(-)$ - $\alpha$-pinene & Culex pipiens pallens & 70.36 & [38] \\
\hline$(-)-\alpha$-pinene & Culex pipiens pallens & 31.98 & [39] \\
\hline$(-)-\alpha$-pinene & Ochlerotatus togoi & 57.93 & [38] \\
\hline
\end{tabular}

The larvicidal activities of E. hieraciifolius and E. valerianifolius essential oils can be attributed to the high concentrations of $\alpha$-pinene and limonene in E. hieraciifolius oil and $\alpha$-pinene, myrcene, and $\beta$-caryophyllene in E. valerianifolius oil. However, synergy between essential oil components may also be important $[49,52]$. Scalerandi and coworkers have demonstrated that Musca domestica preferentially metabolizes the major components in an essential oil while leaving the components of lower concentrations to act as toxicants [53]. 
In order to assess the potential environmental impact of using Erechtites essential oils as a larvicidal control agent, we have carried out lethality assays on non-target aquatic species: the water flea, Daphnia magna Straus (Cladocera: Daphniidae); non-biting midge larvae, Chironomus tentans Fabricius (Diptera: Chironomidae); and zebrafish, Danio rerio Hamilton (Cypriniformes: Cyprinidae) (Table 8).

Table 8. Non-target lethality $\left(\mathrm{LC}_{50}, \mu \mathrm{g} / \mathrm{mL}\right)$ of Erechtites hieraciifolius and Erechtites valerianifolius aerial parts (leaves and stems) essential oils.

\begin{tabular}{|c|c|c|c|c|c|c|}
\hline \multicolumn{7}{|c|}{ Erechtites hieraciifolius } \\
\hline $\begin{array}{l}\text { Non-Target } \\
\text { Species }\end{array}$ & $\begin{array}{l}\text { Treatment } \\
\text { Time }\end{array}$ & $\begin{array}{c}\mathrm{LC}_{50, \mu \mathrm{g} / \mathrm{Ml}^{\mathrm{a}}} \\
\text { (Fiducial } \\
\text { Limits) }\end{array}$ & $\begin{array}{c}\mathrm{LC}_{90}, \mu \mathrm{g} / \mathrm{mL}^{\mathrm{a}} \\
\text { (Fiducial } \\
\text { Limits)) }\end{array}$ & $\begin{array}{l}\text { Regression } \\
\text { Equation }\end{array}$ & $x^{2}$ & $P$ \\
\hline Daphnia magna & $24 \mathrm{~h}$ & $\begin{array}{c}0.931 \\
(0.808-1.035) \\
0.909 \pm 0.169\end{array}$ & $\begin{array}{c}1.531 \\
(1.386-1.767)\end{array}$ & $y=-1.897+0.153 x$ & $8.2 \times 10^{-4}$ & 0.977 \\
\hline Daphnia magna & $48 \mathrm{~h}$ & $\begin{array}{c}0.874 \\
(0.754-0.974) \\
0.864 \pm 0.180\end{array}$ & $\begin{array}{c}1.431 \\
(1.297-1.644)\end{array}$ & $y=-2.011+2.301 x$ & $8.1 \times 10^{-5}$ & 0.993 \\
\hline $\begin{array}{l}\text { Chironomus } \\
\text { tentans }\end{array}$ & $24 \mathrm{~h}$ & $\begin{array}{c}10.01 \\
(9.18-10.90) \\
9.37 \pm 0.57\end{array}$ & $\begin{array}{c}14.73 \\
(13.46-16.71)\end{array}$ & $y=-2.723+0.272 x$ & 0.0037 & 0.951 \\
\hline $\begin{array}{l}\text { Chironomus } \\
\text { tentans }\end{array}$ & $48 \mathrm{~h}$ & $\begin{array}{c}7.81 \\
(6.27-9.03) \\
7.64 \pm 0.51\end{array}$ & $\begin{array}{c}15.42 \\
(13.56-18.73)\end{array}$ & $y=-1.315+0.168 x$ & 0.370 & 0.543 \\
\hline Danio rerio & $24 \mathrm{~h}$ & $\begin{array}{c}12.41 \\
(11.11-13.78) \\
11.21 \pm 1.47\end{array}$ & $\begin{array}{c}21.18 \\
(19.12-24.22)\end{array}$ & $y=-1.897+0.153 x$ & 1.34 & 0.247 \\
\hline Daphnia magna & $24 \mathrm{~h}$ & $\begin{array}{c}0.969 \\
(0.871-1.061) \\
0.937 \pm 0.150\end{array}$ & $\begin{array}{c}1.471 \\
(1.347-1.656)\end{array}$ & $y=-2.478+2.556 x$ & $1.7 \times 10^{-5}$ & 0.997 \\
\hline Daphnia magna & $48 \mathrm{~h}$ & $\begin{array}{c}0.917 \\
(0.837-0.999) \\
0.901 \pm 0.119\end{array}$ & $\begin{array}{c}1.298 \\
(1.190-1.464)\end{array}$ & $y=-3.081+3.361 x$ & 0 & 1.0 \\
\hline $\begin{array}{l}\text { Chironomus } \\
\text { tentans }\end{array}$ & $24 \mathrm{~h}$ & $\begin{array}{c}10.12 \\
(8.85-11.40) \\
10.08 \pm 2.58\end{array}$ & $\begin{array}{c}17.99 \\
(15.97-21.28)\end{array}$ & $y=-1.650+0.163 x$ & 1.98 & 0.159 \\
\hline $\begin{array}{l}\text { Chironomus } \\
\text { tentans }\end{array}$ & $48 \mathrm{~h}$ & $\begin{array}{c}5.63 \\
(2.67-7.47) \\
6.67 \pm 0.81\end{array}$ & $\begin{array}{c}16.31 \\
(14.07-20.35)\end{array}$ & $y=-0.677+0.120 x$ & 2.90 & 0.088 \\
\hline Danio rerio & $24 \mathrm{~h}$ & $\begin{array}{c}18.37 \\
(16.89-20.00) \\
16.75 \pm 1.81\end{array}$ & $\begin{array}{c}27.77 \\
(25.45-31.04)\end{array}$ & $y=-2.505+0.136 x$ & 11.38 & 0.001 \\
\hline
\end{tabular}

a There was no mortality in the DMSO controls; $\mathrm{LC}_{50}$ values in italics are from Reed-Muench analysis.

Unfortunately, the Erechtites essential oils also show toxicity to representative non-target organisms, with $\mathrm{LC}_{50}$ values against the midge larvae (C. tentans) and the zebrafish $(D$. rerio) comparable to those for laboratory-reared mosquito larvae. The small crustacean (D. magna) was particularly susceptible to the Erechtites essential oils. Therefore, care must be taken if these essential oils are to be used in broad applications. Local application of Erechtites essential oils (e.g., urban areas) may prove useful as controls for container-breeding mosquitoes, however.

\section{Conclusions}

Erechtites hieraciifolius and E. valerianifolius are introduced weedy species that grow prolifically in Vietnam, particularly where forests have been cleared; acquisition of abundant quantities of plant material should not be a problem. Mosquito larvicidal screening of these two species indicates good larvicidal activity, which can be attributed to their major components. Thus, this work provides evidence that otherwise noxious introduced weeds might provide low-cost vector control agents to prevent the spread of arboviral infections in Vietnam. 
Author Contributions: Conceptualization, N.H.H. and P.S.; methodology, H.V.H., N.H.H., and P.S.; software, P.S.; validation, H.V.H., N.H.H., P.S., and W.N.S.; formal analysis, W.N.S.; investigation, H.V.H., N.H.H., N.T.H.C., T.A.T., D.N.D., and L.T.H.; resources, N.H.H. and P.S.; data curation, W.N.S.; writing-original draft preparation, W.N.S.; writing—review \& editing, H.V.H., N.H.H., P.S., and W.N.S.; visualization, W.N.S.; supervision, N.H.H. and W.N.S.; project administration, N.H.H.; funding acquisition, N.H.H.

Funding: This research was funded by Duy Tan University.

Acknowledgments: P.S. and W.N.S. participated in this work as part of the activities of the Aromatic Plant Research Center (APRC, https://aromaticplant.org/).

Conflicts of Interest: The authors declare no conflict of interest.

\section{References}

1. Tilak, R.; Ray, S.; Tilak, V.W.; Mukherji, S. Dengue, chikungunya ... and the missing entity-Zika fever: A new emerging threat. Med. J. Armed Forces India 2016, 72, 157-163. [CrossRef] [PubMed]

2. Mayer, S.V.; Tesh, R.B.; Vasilakis, N. The emergence of arthropod-borne viral diseases: A global prospective on dengue, chikungunya and zika fevers. Acta Trop. 2017, 166, 155-163. [CrossRef] [PubMed]

3. Wilder-Smith, A.; Gubler, D.J.; Weaver, S.C.; Monath, T.P.; Heymann, D.L.; Scott, T.W. Epidemic arboviral diseases: Priorities for research and public health. Lancet Infect. Dis. 2017, 17, e101-e106. [CrossRef]

4. Kim Lien, P.T.; Briant, L.; Tang, T.B.; Trang, B.M.; Gavotte, L.; Cornillot, E.; Duoc, V.T.; Duong, T.N.; Frutos, R.; Nga, P.T. Surveillance of dengue and chikungunya infection in Dong Thap, Vietnam: A 13-month study. Asian Pac. J. Trop. Med. 2016, 9, 39-43. [CrossRef] [PubMed]

5. Pham Thi, K.L.; Briant, L.; Gavotte, L.; Labbe, P.; Perriat-Sanguinet, M.; Cornillot, E.; Vu, T.D.; Nguyen, T.Y.; Tran, V.P.; Nguyen, V.S.; et al. Incidence of dengue and chikungunya viruses in mosquitoes and human patients in border provinces of Vietnam. Parasites Vectors 2017, 10, 556. [CrossRef] [PubMed]

6. Bowman, L.R.; Donegan, S.; McCall, P.J. Is dengue vector control deficient in effectiveness or evidence?: Systematic review and meta-analysis. PLoS Negl. Trop. Dis. 2016, 10, e0004551. [CrossRef] [PubMed]

7. Benelli, G. Research in mosquito control: Current challenges for a brighter future. Parasitol. Res. 2015, 114, 2801-2805. [CrossRef]

8. Benelli, G. Plant-borne ovicides in the fight against mosquito vectors of medical and veterinary importance: A systematic review. Parasitol. Res. 2015, 114, 3201-3212. [CrossRef]

9. Masetti, A. The potential use of essential oils against mosquito larvae: A short review. Bull. Insectol. 2016, 69, 307-310.

10. Pavela, R.; Benelli, G. Essential oils as ecofriendly biopesticides? Challenges and constraints. Trends Plant Sci. 2016, 21, 1000-1007. [CrossRef]

11. Barkley, T.M. Erechtites. Available online: http://www.efloras.org/florataxon.aspx?flora_id=1\&taxon_id= 111911 (accessed on 15 November 2018).

12. Auld, B.; Morita, H.; Nicshida, T.; Ito, M.; Michael, P. Shared exotica: Plant invasions of Japan and south eastern Australia. Cunninghamia 2003, 8, 147-152.

13. Wu, S.-H.; Hsieh, C.-F.; Rejmánek, M. Catalogue of the naturalized flora of Taiwan. Taiwania 2004, 49, 16-31.

14. Mito, T.; Uesugi, T. Invasive alien species in Japan: The status quo and the new regulation for prevention of their adverse effects. Glob. Environ. Res. 2004, 8, 171-191.

15. Xu, H.; Qiang, S.; Genovesi, P.; Ding, H.; Wu, J.; Meng, L.; Han, Z.; Miao, J.; Hu, B.; Guo, J.; et al. An inventory of invasive alien species in China. NeoBiota 2012, 15, 1-26. [CrossRef]

16. Celka, Z.; Szkudlarz, P.; Shevera, M.V.; Milicka, N. Morphological variation of Erechtites hieracifolia (L). Raf. ex DC. (Asteraceae) achenes in the zone of the species' geographic range expansion, based on the localities from east-central Europe. Balt. For. 2017, 23, 356-363.

17. Bakar, B.H. Invasive weed species in Malaysian agro-ecosystems: Species impacts and management. Malays. J. Sci. 2004, 23, 1-42.

18. Wu, S.-H.; Sun, H.-T.; Teng, Y.-C.; Rejmánek, M.; Chaw, S.-M.; Yang, T.-Y.A.; Hsieh, C.-F. Patterns of plant invasions in China: Taxonomic, biogeographic, climatic approaches and anthropogenic effects. Biol. Invasions 2010, 12, 2179-2206. [CrossRef]

19. Morton, J.F. Atlas of Medicinal Plants of Middle America, Volume II; Charles C. Thomas: pringfield, IL, USA, 1981. 
20. Arévalo-Lopéz, D.; Nina, N.; Ticona, J.C.; Limachi, I.; Salamanca, E.; Udaeta, E.; Paredes, C.; Espinoza, B.; Serato, A.; Garnica, D.; et al. Leishmanicidal and cytotoxic activity from plants used in Tacana traditional medicine (Bolivia). J. Ethnopharmacol. 2018, 216, 120-133. [CrossRef]

21. Darbyshire, S.J.; Francis, A.; DiTommaso, A.; Clements, D.R. The biology of Canadian weeds. 150 Erechtites hieraciifolius (L.) Raf. ex DC. Can. J. Plant Sci. 2012, 92, 729-746. [CrossRef]

22. Adams, R.P. Identification of Essential Oil Components by Gas Chromatography/Mass Spectrometry, 4th ed.; Allured Publishing: Carol Stream, IL, USA, 2007.

23. Satyal, P. Development of GC-MS Database of Essential Oil Components by the Analysis of Natural Essential Oils and Synthetic Compounds and Discovery of Biologically Active Novel Chemotypes in Essential Oils. Ph.D. Dissertation, University of Alabama in Huntsville, Huntsville, AL, USA, 2015.

24. Finney, D. Probit Analysis, Reissue, Ed. ed; Cambridge University Press: Cambridge, UK, 2009.

25. Reed, L.J.; Muench, H. A simple method of estimating fifty per cent endpoints. Am. J. Hyg. 1938, $27,493-497$.

26. Lemos, T.L.G.; Monte, F.J.Q.; Machado, M.I.L.; Parente, L.L.; Campos, T.A. Essential oil of Erichtites hieracifolia. J. Essent. Oil Res. 1998, 10, 217-218. [CrossRef]

27. Do Amaral, W.; Deschamps, C.; Biasi, L.A.; Bizzo, H.R.; Machado, M.P.; da Silva, L.E. Yield and chemical composition of the essential oil of species of the Asteraceae family from Atlantic Forest, south of Brazil. J. Essent. Oil Res. 2018, 30, 278-284. [CrossRef]

28. Missouri Botanical Garden Tropicos.org. Available online: www.tropicos.org (accessed on 21 January 2019).

29. Sharopov, F.S.; Sulaimonova, V.A.; Setzer, W.N. Composition of the essential oil of Artemisia absinthium from Tajikistan. Rec. Nat. Prod. 2012, 6, 127-134.

30. Alves, N.S.F.; Setzer, W.N.; da Silva, J.K.R. The chemistry and biological activities of Peperomia pellucida (Piperaceae): A critical review. J. Ethnopharmacol. 2019, 232, 90-102. [CrossRef] [PubMed]

31. Lorenzo, D.; Saavedra, G.; Loayza, I.; Dellacassa, E. Composition of the essential oil of Erechtites hieracifolia from Bolivia. Flavour Fragr. J. 2001, 16, 353-355. [CrossRef]

32. Hernández, J.; Bracho, I.; Rojas-Fermin, L.B.; Usubillaga, A.; Carmona, J. Chemical composition of the essential oil of Erechtites valerianaefolia from Mérida, Venezuela. Nat. Prod. Commun. 2013, 8, 1477-1478. [PubMed]

33. Dias, C.N.; Fernandes, D.; Moraes, C. Essential oils and their compounds as Aedes aegypti L. (Diptera: Culicidae) larvicide: Review. Parasitol. Res. 2014, 113, 565-592. [CrossRef]

34. Pavela, R. Essential oils for the development of eco-friendly mosquito larvicides: A review. Ind. Crops Prod. 2015, 76, 174-187. [CrossRef]

35. Amer, A.; Mehlhorn, H. Larvicidal effects of various essential oils against Aedes, Anopheles, and Culex larvae (Diptera, Culicidae). Parasitol. Res. 2006, 99, 466-472. [CrossRef]

36. Pavela, R. Larvicidal property of essential oils against Culex quinquefasciatus Say (Diptera: Culicidae). Ind. Crops Prod. 2009, 30, 311-315. [CrossRef]

37. Benelli, G.; Pavela, R.; Canale, A.; Cianfaglione, K.; Ciaschetti, G.; Conti, F.; Nicoletti, M.; Senthil-Nathan, S.; Mehlhorn, H.; Maggi, F. Acute larvicidal toxicity of five essential oils (Pinus nigra, Hyssopus officinalis, Satureja montana, Aloysia citrodora and Pelargonium graveolens) against the filariasis vector Culex quinquefasciatus: Synergistic and antagonistic effects. Parasitol. Int. 2017, 66, 166-171. [CrossRef] [PubMed]

38. Perumalsamy, H.; Kim, N.-J.; Ahn, Y.-J. Larvicidal activity of compounds isolated from Asarum heterotropoides against Culex pipiens pallens, Aedes aegypti, and Ochlerotatus togoi (Diptera: Culicidae). J. Med. Entomol. 2009, 46, 1420-1423. [CrossRef] [PubMed]

39. Lee, D.C.; Ahn, Y.J. Laboratory and simulated field bioassays to evaluate larvicidal activity of Pinus densiflora hydrodistillate, its constituents and structurally related compounds against Aedes albopictus, Aedes aegypti and Culex pipiens pallens in relation to their inhibitory effects on acetylcholinesterase activity. Insects 2013, 4, 217-229. [PubMed]

40. Govindarajan, M.; Rajeswary, M.; Hoti, S.L.; Bhattacharyya, A.; Benelli, G. Eugenol, $\alpha$-pinene and $\beta$-caryophyllene from Plectranthus barbatus essential oil as eco-friendly larvicides against malaria, dengue and Japanese encephalitis mosquito vectors. Parasitol. Res. 2016, 115, 807-815. [CrossRef] [PubMed]

41. Silva, W.J.; Dória, G.A.A.; Maia, R.T.; Nunes, R.S.; Carvalho, G.A.; Blank, A.F.; Alves, P.B.; Marçal, R.M.; Cavalcanti, S.C.H. Effects of essential oils on Aedes aegypti larvae: Alternatives to environmentally safe insecticides. Bioresour. Technol. 2008, 99, 3251-3255. [CrossRef] [PubMed] 
42. Cheng, S.-S.; Chang, H.-T.; Lin, C.-Y.; Chen, P.-S.; Huang, C.-G.; Chen, W.-J.; Chang, S.-T. Insecticidal activities of leaf and twig essential oils from Clausena excavata against Aedes aegypti and Aedes albopictus larvae. Pest Manag. Sci. 2009, 65, 339-343. [CrossRef]

43. Cheng, S.-S.; Liu, J.-Y.; Huang, C.-G.; Hsui, Y.-R.; Chen, W.-J.; Chang, S.-T. Insecticidal activities of leaf essential oils from Cinnamomum osmophloeum against three mosquito species. Bioresour. Technol. 2009, 100, 457-464. [CrossRef]

44. Santos, S.R.L.; Melo, M.A.; Cardoso, A.V.; Santos, R.L.C.; de Sousa, D.P.; Cavalcanti, S.C.H. Structure-activity relationships of larvicidal monoterpenes and derivatives against Aedes aegypti Linn. Chemosphere 2011, 84, 150-153. [CrossRef]

45. Cheng, S.S.; Lin, C.Y.; Chung, M.J.; Liu, Y.H.; Huang, C.G.; Chang, S.T. Larvicidal activities of wood and leaf essential oils and ethanolic extracts from Cunninghamia konishii Hayata against the dengue mosquitoes. Ind. Crops Prod. 2013, 47, 310-315. [CrossRef]

46. Giatropoulos, A.; Papachristos, D.P.; Kimbaris, A.; Koliopoulos, G.; Polissiou, M.G.; Emmanouel, N.; Michaelakis, A. Evaluation of bioefficacy of three Citrus essential oils against the dengue vector Aedes albopictus (Diptera: Culicidae) in correlation to their components enantiomeric distribution. Parasitol. Res. 2012, 111, 2253-2263. [CrossRef]

47. Seo, S.-M.; Jung, C.-S.; Kang, J.; Lee, H.-R.; Kim, S.-W.; Hyun, J.; Park, I.-K. Larvicidal and acetylcholinesterase inhibitory activities of Apiaceae plant essential oils and their constituents against Aedes albopictus and formulation development. J. Agric. Food Chem. 2015, 63, 9977-9986. [CrossRef] [PubMed]

48. Liu, X.C.; Liu, Q.; Chen, X.B.; Zhou, L.; Liu, Z.L. Larvicidal activity of the essential oil from Tetradium glabrifolium fruits and its constituents against Aedes albopictus. Pest Manag. Sci. 2015, 71, 1582-1586. [CrossRef] [PubMed]

49. Pavela, R. Acute toxicity and synergistic and antagonistic effects of the aromatic compounds of some essential oils against Culex quinquefasciatus Say larvae. Parasitol. Res. 2015, 114, 3835-3853. [CrossRef] [PubMed]

50. Lucia, A.; Gonzalez Audino, P.; Seccacini, E.; Licastro, S.; Zerba, E.; Masuh, H. Larvicidal effect of Eucalyptus grandis essential oil and turpentine and their major components on Aedes aegypti larvae. J. Am. Mosq. Control Assoc. 2007, 23, 299-303. [CrossRef]

51. Traboulsi, A.F.; Taoubi, K.; El-Haj, S.; Bessiere, J.M.; Rammal, S. Insecticidal properties of essential plant oils against the mosquito Culex pipiens molestus (Diptera: Culicidae). Pest Manag. Sci. 2002, 58, 491-495. [CrossRef] [PubMed]

52. Tak, J.H.; Isman, M.B. Penetration-enhancement underlies synergy of plant essential oil terpenoids as insecticides in the cabbage looper, Trichoplusia ni. Sci. Rep. 2017, 7, 42432. [CrossRef] [PubMed]

53. Scalerandi, E.; Flores, G.A.; Palacio, M.; Defagó, M.T.; Carpinella, M.C.; Valladares, G.; Bertoni, A.; Palacios, S.M. Understanding synergistic toxicity of terpenes as insecticides: Contribution of metabolic detoxification in Musca domestica. Front. Plant Sci. 2018, 9, 1579. [CrossRef]

(C) 2019 by the authors. Licensee MDPI, Basel, Switzerland. This article is an open access article distributed under the terms and conditions of the Creative Commons Attribution (CC BY) license (http://creativecommons.org/licenses/by/4.0/). 